\title{
SADDLE-NODE BIFURCATIONS IN CLASSICAL AND MEMRISTIVE CIRCUITS*
}

\author{
IGNACIO GARCÍA DE LA VEGA and RICARDO RIAZA
}

\begin{abstract}
This paper addresses a systematic characterization of saddle-node bifurcations in nonlinear electrical and electronic circuits. Our approach is a circuit-theoretic one, meaning that the bifurcation is analyzed in terms of the devices' characteristics and the graph-theoretic properties of the digraph underlying the circuit. The analysis is based on a reformulation of independent interest of the saddle-node theorem of Sotomayor for semiexplicit index one differential-algebraic equations (DAEs), which define the natural context to set up nonlinear circuit models. The bifurcation is addressed not only for classical circuits, but also for circuits with memristors. The presence of this device systematically leads to non-isolated equilibria, and in this context the saddle-node bifurcation is shown to yield a bifurcation of manifolds of equilibria; in cases with a single memristor, this phenomenon describes the splitting of a line of equilibria into two, with different stability properties.
\end{abstract}

Keywords: Saddle-node bifurcation, equilibrium point, differential-algebraic equation, matrix pencil, graph, circuit model, memristor.

\section{Introduction}

Bifurcations of equilibria are a key element in the analysis of the local behavior of nonlinear dynamical systems. Broadly speaking, in a parameterized setting a bifurcation of an equilibrium point describes a qualitative change in the local phase portrait of the system when the parameter undergoes a critical value. In nonlinear circuit theory, such bifurcations correspond to qualitative changes in the local dynamics around a given operating point, and their characterization is important since they may result in a loss of stability; they may also be relevant as an auxiliary tool in the analysis of other qualitative phenomena involving e.g. periodic solutions. Many qualitative properties of nonlinear electrical and electronic circuits have been addressed in the literature; find references in the books [Choma, 1985; Chua et al., 1987; Fosséprez, 1992; Hasler and Neirynck, 1986; Riaza, 2008]. Milestones in this field are of course Van der Pol's and Chua's circuits; find the 1920 papers by Van der Pol in [Van der Pol, 1920] and exhaustive analyses of Chua's circuit in [Fortuna et al., 2009; Madan, 1993] and references therein. Other key papers addressing qualitative 
properties in the context of classical nonlinear circuits are [Brayton and Moser, 1964; Chua, 1980; Mees and Chua, 1979; Smale, 1972]; we use the term "classical" to denote circuits without memristive devices.

The main local bifurcation of equilibria is the so-called saddle-node bifurcation, in which an equilibrium point depicting a simple zero eigenvalue (in the linearization of the vector field defining the dynamics) at a critical parameter value bifurcates into two different equilibria as the parameter varies. These two equilibria differ in the sign of a real eigenvalue and, in the particular case of two-dimensional dynamics, they are necessarily a saddle point (that is, an equilibrium point with two nonzero, real eigenvalues with opposite sign) and a node (namely an equilibrium with two nonzero, real eigenvalues with the same sign). The latter may be stable or unstable, depending on the sign of the eigenvalue which stays away from the origin. Moreover, this behavior is generic in the set of smooth, one-parameter vector fields with an equilibrium having a simple zero eigenvalue, according to the fundamental work of Sotomayor [Sotomayor, 1973a,b]. Find detailed discussions in [Guckenheimer and Holmes, 1983; Perko, 1991]. Saddle-node bifurcation points are a particular instance of so-called turning points, which are important in numerical continuation theory [Allgower and Georg, 2003; Goovaerts, 2000]; preliminary results of our research, regarding quadratic turning points, can be found in [García de la Vega and Riaza, 2014]. In this context, the goal of the present work is to address systematically the existence of saddle-node bifurcations in dynamical systems modelling nonlinear circuits, both in a classical setting and also in the presence of memristors. The latter device has had a great impact in electrical and electronic engineering since the announcement of the design of a nanoscale memristor in [Strukov et al., 2008] (see also [Chua, 1971; Kavehei et al., 2010; Pershin and Di Ventra, 2011; Tetzlaff, 2014] and additional references in Section 5), and our results should be also relevant in this field of active research.

Time-domain descriptions of electrical and electronic circuits are usually defined in terms of so-called semistate models based on differential-algebraic equations (DAEs). This is due to the fact that deriving state models based on explicit ODEs is usually not feasible in practice: additionally, from an analytical point of view, a state-space derivation often involves strong theoretical assumptions which are not really needed in a qualitative analysis such as the one addressed in the present work. For this reason, our approach necessarily involves a reformulation of Sotomayor conditions in DAE terms. General background on DAE theory can be found in [Brenan et al., 1996; Kunkel and Mehrmann, 2006; Lamour et al., 2013; Rabier and Rheinboldt, 2002; Riaza, 2008], and DAE-based circuit modelling is extensively discussed in [EstévezSchwarz and Tischendorf, 2000; Günther and Feldmann, 1995; Günther and Rentrop, 1996; Lamour et al., 2013; Riaza, 2008; Tischendorf, 1999, 2003]. DAEs are nowadays pervasive in circuit simulation programs, such as SPICE, which set up the circuit equations using Modified Nodal Analysis (MNA) techniques.

The DAE approach makes it possible to address the saddle-node bifurcation in circuit-theoretic terms, at least for a broad class of nonlinear circuits. This means that the conditions characterizing the bifurcation can be formulated in terms of both the electrical features of the circuit devices (passivity, linearity, coupling effects, etc.) and the topological properties of the digraph underlying the circuit. The origin of this graph-theoretic or topological approach can be traced back to the state-space formulation problem in the 1960s and 1970s [Brown, 1963; Bryant and Tow, 1972], and has been successfully applied to qualitative analyses of nonlinear circuits [Chua, 1980; Fosséprez, 1992; Haggman and Bryant, 1984, 1986] and to the characterization of the index of DAE circuit models [Estévez-Schwarz and Tischendorf, 2000; Günther and Feldmann, 1995; Günther and Rentrop, 1996; Riaza, 2008; Tischendorf, 1999, 2003].

The paper is structured as follows. We compile background material in Section 2. In Section 3 we extend the saddle-node bifurcation theorem to the context of semiexplicit index one DAEs; although this result is mainly intended for later use, it is also believed to be of independent interest. Our main goal is addressed in Section 4, where we present a graph-theoretic characterization of the saddle-node bifurcation for a broad family of classical circuits. We then extend this approach to the context of memristive circuits in Section 5; the systematic presence of manifolds of non-isolated equilibria transforms the saddle-node bifurcation into a bifurcation of manifolds of equilibria, or lines of equilibria in the presence of only one memristor. Finally, Section 6 compiles some concluding remarks. 


\section{Background}

\subsection{The saddle-node bifurcation theorem for explicit ODEs}

The reader is referred to [Guckenheimer and Holmes, 1983; Perko, 1991] and to the original papers [Sotomayor, 1973a,b] for detailed discussions of the saddle-node bifurcation theorem. As shown by Sotomayor, given a parameterized explicit ODE

$$
x^{\prime}=f(x, \mu),
$$

with $x \in \mathbb{R}^{r}$ and $f \in C^{\infty}\left(\mathbb{R}^{r} \times \mathbb{R}, \mathbb{R}^{r}\right)$. Note that smoothness requirements can be relaxed if needed, and that the analysis can be carried out in an open subset of $\mathbb{R}^{r}$ or, locally, in terms of germs; the setting above is kept deliberately simple in order to make the results accessible to a broader audience. An equilibrium point $\left(x^{*}, \mu^{*}\right)$ of (1) yields a saddle-node bifurcation if the conditions below are met. We use a subscript to denote partial differentiation, that is, $f_{x}$ and $f_{\mu}$ are the matrices of partial derivatives of $f$ with respect to $x$ and $\mu$, respectively.

(1) The matrix $f_{x}\left(x^{*}, \mu^{*}\right)$ has a simple zero eigenvalue, and the remaining eigenvalues are away from the imaginary axis.

(2) If $w$ is a left eigenvector of the null eigenvalue of $f_{x}\left(x^{*}, \mu^{*}\right)$, then $w^{T} f_{\mu}\left(x^{*}, \mu^{*}\right) \neq 0$.

(3) If $v$ is a right eigenvector of the null eigenvalue of $f_{x}\left(x^{*}, \mu^{*}\right)$, then $w^{T} f_{x x}\left(x^{*}, \mu^{*}\right)(v, v) \neq 0$.

Note that $v$ and $w$ are simply non-vanishing vectors in the kernel of $f_{x}\left(x^{*}, \mu^{*}\right)$ and $\left(f_{x}\left(x^{*}, \mu^{*}\right)\right)^{\top}$, respectively. Under conditions 1-3 above, a smooth curve of equilibria passes through $\left(x^{*}, \mu^{*}\right)$, in a way such that for $\mu<\mu^{*}$ (or, conversely, for $\mu>\mu^{*}$ ) system (1) has no equilibria, whereas for $\mu>\mu^{*}$ (resp. $\mu<\mu^{*}$ ) there are two equilibrium points. Both equilibria are hyperbolic, that is, all eigenvalues have non-vanishing real parts, and the number of eigenvalues with positive (or negative) real part differ in one from one equilibrium to another; this means that one real eigenvalue crosses the origin and changes sign at $\left(x^{*}, \mu^{*}\right)$ as we move along the equilibrium branch.

For later use, we note that condition 1 implies $\operatorname{rk} f_{x}\left(x^{*}, \mu^{*}\right)=r-1$, and this allows for different restatements of conditions 2 and 3 . Specifically, condition 2 above can be equivalently recast as

$$
f_{\mu}\left(x^{*}, \mu^{*}\right) \notin \operatorname{im} f_{x}\left(x^{*}, \mu^{*}\right),
$$

and also as the maximal rank condition

$$
\operatorname{rk} f^{\prime}\left(x^{*}, \mu^{*}\right)=r,
$$

where $f^{\prime}$ is the full matrix of partial derivatives of $f$, that is, $f^{\prime}=\left(\begin{array}{ll}f_{x} & f_{\mu}\end{array}\right)$. From (3) it follows that the equation $f(x, \mu)=0$ describes indeed a curve of equilibria locally around $\left(x^{*}, \mu^{*}\right)$, and one can check that this curve is tangent to the hyperplane $\mu=\mu^{*}$.

Analogously, condition 3 can be rewritten as

$$
f_{x x}\left(x^{*}, \mu^{*}\right)(v, v) \notin \operatorname{im} f_{x}\left(x^{*}, \mu^{*}\right) .
$$

Another reformulation of this condition comes from singularity theory. Indeed, $x^{*}$ is a fold (see e.g. [Golubitsky and Guillemin, 1973; Martinet, 1982]) and (4) expresses, within the hyperplane $\mu=\mu^{*}$, the transversal intersection at $x=x^{*}$ of the so-called singular manifold, defined by det $f_{x}\left(x, \mu^{*}\right)=0$, and the space ker $f_{x}\left(x^{*}, \mu^{*}\right)$. This means that condition 3 can be also expressed as

$$
\left(\operatorname{det} f_{x}\right)_{x}\left(x^{*}, \mu^{*}\right) v \neq 0,
$$

for some (hence any) $v \in \operatorname{ker} f_{x}\left(x^{*}, \mu^{*}\right)-\{0\}$. This reformulation makes it also possible to express condition 3 as

$$
\operatorname{rk}\left(\begin{array}{c}
f_{x}\left(x^{*}, \mu^{*}\right) \\
\left(\operatorname{det} f_{x}\right)_{x}\left(x^{*}, \mu^{*}\right)
\end{array}\right)=r
$$

At several points in our analysis we will make use of the alternative descriptions of conditions 2 and 3 above given by (2)-(6). 


\subsection{Elementary properties of digraphs}

In Section 4 we will make systematic use of several properties of directed graphs (digraphs); these properties are compiled below. Find more detailed discussions of this material in [Andrásfai, 1991; Bollobás, 1998; Diestel, 2010; Riaza, 2008].

Kirchhoff laws will be described in terms of the so-called reduced cutset and loop (or cycle) matrices. A subset $K$ of the set of branches of a digraph is a cutset if the removal of $K$ increases the number of connected components of the digraph, and this set is minimal with respect to this property, that is, the removal of any proper subset of $K$ does not increase this number. A cutset is oriented just by directing it from one of the connected components resulting from the cutset deletion towards the other. A loop or cycle denotes the branches in a closed path without self-intersections, and an orientation is defined after choosing a sense in which this path is traversed.

After choosing an orientation for every cutset, the entries of the so-called cutset matrix $\bar{Q}=\left(q_{i j}\right)$ are given by

$$
q_{i j}=\left\{\begin{aligned}
1 & \text { if branch } j \text { is in cutset } i \text { with the same orientation } \\
-1 & \text { if branch } j \text { is in cutset } i \text { with the opposite orientation } \\
0 & \text { if branch } j \text { is not in cutset } i .
\end{aligned}\right.
$$

This matrix is known to have rank $n-k$, provided that $n$ and $k$ are the number of nodes and connected components. Any set of $(n-k)$ linearly independent rows of $\bar{Q}$ yields a reduced cutset matrix $Q \in \mathbb{R}^{(n-k) \times m}$, where $m$ is the number of branches.

Similarly, by choosing an orientation for every loop one gets the loop or cycle matrix $\bar{B}=\left(b_{i j}\right)$ with entries

$$
b_{i j}=\left\{\begin{aligned}
1 & \text { if branch } j \text { is in loop } i \text { with the same orientation } \\
-1 & \text { if branch } j \text { is in loop } i \text { with the opposite orientation } \\
0 & \text { if branch } j \text { is not in loop } i .
\end{aligned}\right.
$$

The rank of this matrix equals the cyclomatic number $m-n+k$, and the choice of any set of $(m-n+k)$ linearly independent rows of $\bar{B}$ defines a reduced loop matrix $B \in \mathbb{R}^{(m-n+k) \times m}$.

The rows of the cutset and loop matrices define the so-called cut space and cycle space, respectively, and choosing $n-k$ and $m-n+k$ rows in the corresponding matrices amount to choosing two bases in these spaces, which can be therefore described as $\operatorname{im} Q^{\top}$ (cut space) and $\operatorname{im} B^{\top}$ (cycle space). Details, including also Lemma 1, can be found e.g. in [Bollobás, 1998].

Lemma 1. The cut- and cycle-spaces are orthogonal to each other.

Using the identity $\left(\operatorname{im} M^{\top}\right)^{\perp}=\operatorname{ker} M$ (holding for any matrix $M$ ) this property can be expressed as $\operatorname{im} B^{\top}=\operatorname{ker} Q$ and $\operatorname{im} Q^{\top}=\operatorname{ker} B$. This implies $Q B^{\top}=0$ and $B Q^{\top}=0$.

By a $K$-loop (resp. $K$-cutset) we mean a loop (resp. cutset) defined only by elements of a given set of branches $K$. The existence of $K$-loops and $K$-cutsets in a given digraph can be described in terms of the matrices $Q$ and $B$, as stated below. Find details e.g. in [Andrásfai, 1991; Bollobás, 1998; Riaza, 2008]. By $Q_{K}$ (resp. $B_{K}$ ) we mean the submatrix of $Q$ (resp. $B$ ) defined by the columns which correspond to branches in $K$.

Lemma 2. A set $K$ of branches of a given digraph does not contain loops if and only if the identity $\operatorname{ker} Q_{K}=\{0\}$ holds. It does not contain cutsets if and only if $\operatorname{ker} B_{K}=\{0\}$.

Moreover, a non-vanishing entry in the position corresponding to a given branch $e$ within a vector of $\operatorname{ker} Q$ (resp. $\operatorname{ker} B$ ) indicates the existence of a loop (resp. a cutset) which includes $e$. From this remark and Lemma 2 one easily gets the following.

Corollary 1. A branch e forms a loop (resp. a cutset) together with some branches from a given set $K$ (not including e) if and only if $Q_{e} \in \operatorname{im} Q_{K}$ (resp. $B_{e} \in \operatorname{im} B_{K}$ ).

Indeed, the existence of such a loop indicates that there is a vector $\left(v_{a} v_{b}\right)$ in $\operatorname{ker}\left(Q_{K} Q_{e}\right)$ with $v_{b} \in \mathbb{R}-\{0\}$. This yields $Q_{e}=-\frac{1}{v_{b}} Q_{K} v_{a}$. The same holds for cutsets. 
We will also make use of the following result. Its proof is straightforward and can be found in [Riaza, 2012].

Lemma 3. Split the branches of a given digraph in four pairwise disjoint sets $K_{1}, K_{2}, K_{3}, K_{4}$, and denote by $B_{i}, Q_{i}$ the submatrices of $B$ and $Q$ defined by the columns which correspond to branches in $K_{i}$. Assume that $P$ is a positive definite matrix and write

$$
M=\left(\begin{array}{ccc}
B_{1} & 0 & B_{3} \\
0 & Q_{2} & Q_{3} P
\end{array}\right)
$$

Then the identity $\operatorname{ker} M=\operatorname{ker} B_{1} \times \operatorname{ker} Q_{2} \times\{0\}$ holds. In particular, the kernel of $M$ is trivial if and only if the digraph has neither $K_{1}$-cutsets nor $K_{2}$-loops.

With $K_{3}=\{e\}, K_{4}=\emptyset$ and $P=(1)$, from Lemma 2 (or Corollary 1 ) and Lemma 3 one gets the following result, which will also be used in Section 4 .

Corollary 2. Split the branches of a given digraph in three pairwise disjoint sets $K_{1}, K_{2}$ and $K_{3}=\{e\}$. If e forms a cutset (resp. a loop) with some branches from $K_{1}$, then it does not form a loop (resp. a cutset) with branches from $K_{2}$.

This is actually part of the colored branch theorem [Minty, 1960; Vandewalle and Chua, 1980] according to which, in a three-color graph with just one blue branch, this branch either forms a cutset exclusively with green branches or a loop exclusively with red branches, but not both. We will only use the latter (that is, the fact that one configuration precludes the other), as stated in Corollary 2. Its proof is very simple; just notice that a cutset (resp. a loop) including $e$ and some branches from $K_{1}$ (resp. $K_{2}$ ) yields a vector in $\operatorname{ker}\left(B_{1} B_{3}\right)$ (resp. $\left.\operatorname{ker}\left(Q_{2} Q_{3}\right)\right)$; the last entries of both vectors do not vanish and rescaling one of them we would construct a vector in $\operatorname{ker} M$ but not in $\operatorname{ker} B_{1} \times \operatorname{ker} Q_{2} \times\{0\}$, against Lemma 3 .

\subsection{Schur complements}

At several points in our analysis we will make use of the notion of the Schur complement [Horn and Johnson, 1985] of a square submatrix $D$ of a given matrix

$$
M=\left(\begin{array}{cc}
A & B \\
C & D
\end{array}\right)
$$

with $M$ not necessarily square. If $D$ is non-singular, the Schur complement of $D$ in $M$ is defined as

$$
(M / D)=A-B D^{-1} C .
$$

Lemma 4. Assume that the blocks in the matrix $M$ in (7) have dimensions $A \in \mathbb{R}^{r \times q}, B \in \mathbb{R}^{r \times p}, C \in \mathbb{R}^{p \times q}$, $D \in \mathbb{R}^{p \times p}$, and that $D$ is non-singular. Then

$$
\operatorname{rk} M=\operatorname{rk}(M / D)+p .
$$

In particular, if $A$ (and therefore $M$ ) is also square (i.e. if $r=q$ ), then

$$
\operatorname{det} M=\operatorname{det}(M / D) \operatorname{det} D \text {. }
$$

Both claims follow immediately from the identity

$$
\left(\begin{array}{cc}
I & -B D^{-1} \\
0 & I
\end{array}\right)\left(\begin{array}{ll}
A & B \\
C & D
\end{array}\right)\left(\begin{array}{cc}
I & 0 \\
-D^{-1} C & I
\end{array}\right)=\left(\begin{array}{cc}
A-B D^{-1} C & 0 \\
0 & D
\end{array}\right)
$$

Recall that the corank of a matrix $M \in \mathbb{R}^{m \times n}$ is $m-\operatorname{rk} M$ (that is, the codimension of the image space) and the nullity is $n-\operatorname{rk} M$ (namely, the dimension of the kernel). The rank identity in Lemma 4 says that the Schur reduction defining $(M / D)$ preserves the rank deficiency of $M$ which, more precisely, stands for the corank if $m \leq n$ or the nullity if $m \geq n$. It is easy to check that both the corank and the nullity of $M$ equal those of the Schur complement $(M / D)$. 


\section{Saddle-node bifurcations in semiexplicit index one DAEs}

We now address the problem of unfolding the saddle-node bifurcation theorem to the setting of semiexplicit DAEs, focusing on index one problems. This is a step (of independent interest) towards our main goal which is to address saddle-node bifurcations in DAE circuit models. Note that the literature on bifurcation theory for DAEs has mainly focused on so-called singularity-induced bifurcations, stemming from the work of Venkatasubramanian et al. [Venkatasubramanian, 1992; Venkatasubramanian et al., 1995]; see also [Beardmore, 2001; Llibre et al., 2002; Riaza, 2010]. A reformulation of the Hopf bifurcation theorem for DAEs can be found in [Rabier and Rheinboldt, 2002].

In this direction, consider a parameterized semiexplicit differential-algebraic equation

$$
\begin{aligned}
x^{\prime} & =h(x, y, \mu) \\
0 & =g(x, y, \mu),
\end{aligned}
$$

with $h \in C^{\infty}\left(\mathbb{R}^{r} \times \mathbb{R}^{p} \times \mathbb{R}, \mathbb{R}^{r}\right), g \in C^{\infty}\left(\mathbb{R}^{r} \times \mathbb{R}^{p} \times \mathbb{R}, \mathbb{R}^{p}\right)$; as in Subsection 2.1 above, the $C^{\infty}$-smoothness assumption can be relaxed if necessary. We will focus on an equilibrium point of (9), that is, a point $\left(x^{*}, y^{*}, \mu^{*}\right)$ which annihilates the right-hand sides of both (9a) and (9b).

We will work under a (local) index one assumption: this means that the matrix of partial derivatives $g_{y}\left(x^{*}, y^{*}, \mu^{*}\right)$ is assumed to be non-singular [Brenan et al., 1996; Kunkel and Mehrmann, 2006; Lamour et al., 2013; Rabier and Rheinboldt, 2002; Riaza, 2008]. This implies, in light of the implicit function theorem, that the manifold $g(x, y, \mu)=0$ can be locally described as

$$
y=\psi(x, \mu)
$$

for a (locally defined) map $\psi(x, \mu)$. The local dynamical behavior of the DAE (9) can be hence described in terms of the so-called reduced $O D E$

$$
x^{\prime}=f(x, \mu)=h(x, \psi(x, \mu), \mu) .
$$

Additionally, the implicit function theorem yields the expression $\psi_{x}=-\left(g_{y}\right)^{-1} g_{x}$ for the partial derivatives, and therefore

$$
f_{x}=h_{x}-h_{y}\left(g_{y}\right)^{-1} g_{x}
$$

the left-hand side holding for $(x, \mu)$ on a neighborhood of $\left(x^{*}, \mu^{*}\right)$, and the right-hand side being evaluated at $(x, \psi(x, \mu), \mu)$.

Locally, equilibria $(x, y, \mu)$ of the DAE $(9)$ are in one-to-one correspondence with equilibrium points $(x, \mu)$ of the reduction (11) via the relation $y=\psi(x, \mu)$. Therefore, a saddle-node bifurcation for the DAE (9) will occur at a given equilibrium $\left(x^{*}, y^{*}, \mu^{*}\right)$ if and only if $\left(x^{*}, \mu^{*}\right)$ is a saddle-node bifurcation point for the reduction (11). For this reason, driving the saddle-node bifurcation theorem to the context of semiexplicit index requires a reformulation of the conditions 1-3 in Subsection 2.1 in terms of the maps $h$ and $g$ arising in (9). This task is performed in Theorem 1 below.

In this regard, notice that linearization properties of DAEs of the form (9) are better framed in the context of matrix pencil theory [Gantmacher, 1959] and, accordingly, condition 1 in Subsection 2.1 will be restated in matrix pencil terms within Theorem 1 . Specifically, linear stability properties of an equilibrium $\left(x^{*}, \mu^{*}\right)$ of the reduction (11) can be examined in terms of the matrix pencil $\lambda A-B^{*}$, with

$$
A=\left(\begin{array}{ll}
I & 0 \\
0 & 0
\end{array}\right), B=\left(\begin{array}{cc}
h_{x} & h_{y} \\
g_{x} & g_{y}
\end{array}\right), B^{*}=B\left(x^{*}, y^{*}, \mu^{*}\right),
$$

where the identity block in the first matrix has order $r \times r$, and $y^{*}=\psi\left(x^{*}, \mu^{*}\right)$. If $g_{y}\left(x^{*}, y^{*}, \mu^{*}\right)$ is nonsingular, then the matrix pencil is regular with Kronecker index one (find details in [Rabier and Rheinboldt, 2002; Riaza, 2008]), and the eigenvalues of the pencil are the (complex) values of $\lambda$ which make $\lambda A-B^{*}$ a singular matrix.

Theorem 1. Consider an equilibrium $\left(x^{*}, y^{*}, \mu^{*}\right)$ of the DAE (9), and assume that the matrix of partial derivatives $g_{y}\left(x^{*}, y^{*}, \mu^{*}\right)$ is non-singular. Let the following conditions be met. 
(1) The matrix pencil $\lambda A-B^{*}$, with $A, B^{*}$ given in (13), has a simple zero eigenvalue, and the remaining eigenvalues are away from the imaginary axis.

(2) The matrix

$$
\hat{B}=\left(\begin{array}{lll}
h_{x} & h_{y} & h_{\mu} \\
g_{x} & g_{y} & g_{\mu}
\end{array}\right)
$$

has maximal rank $r+p$ at $\left(x^{*}, y^{*}, \mu^{*}\right)$.

(3) The matrix

$$
\tilde{B}=\left(\begin{array}{cc}
h_{x} & h_{y} \\
g_{x} & g_{y} \\
(\operatorname{det} B)_{x} & (\operatorname{det} B)_{y}
\end{array}\right)
$$

has maximal rank $r+p$ at $\left(x^{*}, y^{*}, \mu^{*}\right)$.

Then the DAE (9) undergoes a saddle-node bifurcation at $\left(x^{*}, y^{*}, \mu^{*}\right)$; that is, it exhibits a smooth curve of equilibria passing through $\left(x^{*}, y^{*}, \mu^{*}\right)$, in a way such that either for $\mu<\mu^{*}$ or, respectively, for $\mu>\mu^{*}$, the $D A E$ has no equilibria, whereas for $\mu>\mu^{*}$ (resp. for $\mu<\mu^{*}$ ) it has two hyperbolic equilibria; the number of eigenvalues with positive (or negative) real part differ in one from one equilibrium to another.

Proof. As indicated above, we need to check that conditions 1-3 in the statement of Theorem 1 imply that conditions 1-3 in Subsection 2.1 hold for (11).

Note first that the eigenvalues of the matrix pencil $\lambda A-B^{*}$ are the values of $\lambda$ which make the matrix

$$
\lambda\left(\begin{array}{ll}
I & 0 \\
0 & 0
\end{array}\right)-\left(\begin{array}{ll}
h_{x} & h_{y} \\
g_{x} & g_{y}
\end{array}\right)=\left(\begin{array}{cc}
\lambda I-h_{x} & -h_{y} \\
-g_{x} & -g_{y}
\end{array}\right)
$$

singular at $\left(x^{*}, y^{*}, \mu^{*}\right)$. The matrix $g_{y}$ is non-singular at $\left(x^{*}, y^{*}, \mu^{*}\right)$ and therefore the matrix in the righthand side of (16) is singular if and only if so it is its Schur complement (cf. Lemma 4), that is

$$
\lambda I-h_{x}+h_{y}\left(g_{y}\right)^{-1} g_{x}=\lambda I-\left(h_{x}-h_{y}\left(g_{y}\right)^{-1} g_{x}\right),
$$

evaluated again at $\left(x^{*}, y^{*}, \mu^{*}\right)$. According to (12), the values of $\lambda$ which make (17) singular at $\left(x^{*}, y^{*}, \mu^{*}\right)$ are exactly the eigenvalues of $f_{x}\left(x^{*}, \mu^{*}\right)$, and therefore the assumptions that the null eigenvalue is simple and that the remaining ones have non-zero real parts are transferred to (11), so that condition 1 in Subsection 2.1 does hold for this reduced ODE.

Condition 2 is directly transferred to the reduced ODE (11), since the Schur complement of $g_{y}\left(x^{*}, y^{*}, \mu^{*}\right)$ in $\hat{B}$ is

$$
\left(h_{x}-h_{y}\left(g_{y}\right)^{-1} g_{x} h_{\mu}-h_{y}\left(g_{y}\right)^{-1} g_{\mu}\right)
$$

all derivatives being evaluated at $\left(x^{*}, y^{*}, \mu^{*}\right)$. Because of the implicit function theorem, the matrix in (18) is nothing but the derivative $\left(f_{x} f_{\mu}\right)$ at $\left(x^{*}, \mu^{*}\right)$, and therefore the maximal rank condition on (14) yields (3), which is in turn equivalent to condition 2 in Subsection 2.1, as we aimed to show.

Analogously, the Schur complement of $g_{y}\left(x^{*}, y^{*}, \mu^{*}\right)$ in $\tilde{B}$ is

$$
\left(\begin{array}{c}
h_{x}-h_{y}\left(g_{y}\right)^{-1} g_{x} \\
(\operatorname{det} B)_{x}-(\operatorname{det} B)_{y}\left(g_{y}\right)^{-1} g_{x}
\end{array}\right) \text {, }
$$

derivatives being again evaluated at $\left(x^{*}, y^{*}, \mu^{*}\right)$. As before, the maximal rank condition on (15) is transferred to (19). But the problem is now slightly more involved, since the derivatives involved in the last row of (19) are those of $\operatorname{det} B$ and not of $\operatorname{det} f_{x}$, with $f$ coming from (11). In this case we make use of the determinantal formula within Lemma 4, according to which

$$
\operatorname{det} B=\operatorname{det} g_{y} \operatorname{det}\left(h_{x}-h_{y}\left(g_{y}\right)^{-1} g_{x}\right)
$$


at a generic $(x, y, \mu)$ near $\left(x^{*}, y^{*}, \mu^{*}\right)$. Writing $y=\psi(x, \mu)$ we get, in particular,

$$
\operatorname{det} B(x, \psi(x, \mu), \mu)=\operatorname{det} g_{y}(x, \psi(x, \mu), \mu) \operatorname{det} f_{x}(x, \mu) \text {, }
$$

on the manifold $g=0$. By differentiating the product (20) with respect to $x$, and noting that $f_{x}$ is a singular matrix at $\left(x^{*}, \mu^{*}\right)$ (so that $\operatorname{det} f_{x}\left(x^{*}, \mu^{*}\right)=0$ ) because it has a null eigenvalue, we get

$$
\left((\operatorname{det} B)_{x}-(\operatorname{det} B)_{y}\left(g_{y}\right)^{-1} g_{x}\right)\left(x^{*}, y^{*}, \mu^{*}\right)=\operatorname{det} g_{y}\left(x^{*}, y^{*}, \mu^{*}\right)\left(\operatorname{det} f_{x}\right)_{x}\left(x^{*}, \mu^{*}\right),
$$

with $\operatorname{det} g_{y}\left(x^{*}, y^{*}, \mu^{*}\right) \neq 0$ by hypothesis. This means that the matrix (19) reads as

$$
\left(\begin{array}{lc}
I & 0 \\
0 & \operatorname{det} g_{y}\left(x^{*}, y^{*}, \mu^{*}\right)
\end{array}\right)\left(\begin{array}{c}
f_{x}\left(x^{*}, \mu^{*}\right) \\
\left(\operatorname{det} f_{x}\right)_{x}\left(x^{*}, \mu^{*}\right)
\end{array}\right),
$$

and therefore the maximal rank condition on (19) is transferred to the second factor in (22). This is exactly the matrix arising in the reformulation (6) of condition 3 in Subsection 2.1, so that this condition holds for the reduced ODE (11) and this completes the proof of Theorem 1.

This result will make it possible to perform a graph-theoretic analysis of the saddle-node bifurcation for DAE circuit models, as detailed in Section 4.

\section{Saddle-node bifurcations in classical nonlinear circuits}

As indicated in the Introduction, our main goal in this paper is to present a systematic circuit-theoretic analysis of saddle-node bifurcations in nonlinear circuits. This means that we look for conditions which can be stated, and verified in practice, in terms of the devices' characteristics and the graph-theoretic properties of the circuit. This can be done for a broad family of circuits in the terms detailed in Theorem 2 below.

Very close to our approach is the DAE index analysis of nonlinear circuit models carried out in [Estévez-Schwarz and Tischendorf, 2000; Günther and Feldmann, 1995; Günther and Rentrop, 1996; Riaza, 2008; Tischendorf, 1999, 2003], and the qualitative properties examined e.g. in [Chua, 1980; Haggman and Bryant, 1986; Riaza, 2008; Riaza and Tischendorf, 2007]. Specifically, a graph-theoretic index analysis of DAE circuit models under passivity assumptions was developed in [Estévez-Schwarz and Tischendorf, 2000; Tischendorf, 1999, 2003]. In these references, provided that the circuit conductance, capacitance and inductance matrices are everywhere positive definite (that is, assuming the circuit to be strictly locally passive), it is shown that the absence of VC-loops (loops defined by voltage sources and/or capacitors) and IL-cutsets (cutsets formed only by current sources and/or inductors) yields an index-one configuration in nodal analysis models; the same property holds for other families of circuit models (a detailed discussion can be found in [Riaza, 2008]). From a qualitative point of view, it is well-known and can be traced back at least to [Haggman and Bryant, 1986] the property that VL-loops and/or IC-cutsets are responsible for the presence of null eigenvalues in the linearized circuit dynamics. In the absence of these configurations, asymptotic stability of equilibria follows from a strict passivity assumption [Riaza and Tischendorf, 2007].

No changes of stability (in particular, no saddle-node bifurcations) can be then expected in a strictly passive setting. Therefore, we extend the approach in the aforementioned references by allowing a device (specifically, a nonlinear resistor) to become locally active beyond a given operating point. We will assume this device to be governed by a characteristic of the form $i_{n}=\zeta\left(v_{n}\right)$ (we use the subscript $n$, from nonpassive, to distinguish this resistor from the remaining ones, which as usual in this setting will be denoted with a subscript $g$ ) and will work locally around a point where $\zeta^{\prime}\left(v_{n}^{*}\right)=0$ and $\zeta^{\prime \prime}\left(v_{n}^{*}\right) \neq 0$. This means that the characteristic becomes locally active (that is, $\zeta^{\prime}\left(v_{n}\right)<0$ ) either for $v_{n}<v_{n}^{*}$ (if $\left.\zeta^{\prime \prime}\left(v_{n}^{*}\right)>0\right)$ or for $v_{n}>v_{n}^{*}$ (if $\zeta^{\prime \prime}\left(v_{n}^{*}\right)<0$ ). A tunnel diode is a classical example of this behavior, $v_{n}^{*}$ being anyone of the two critical values which delimit the tunnel effect region.

Furthermore, we will assume that the bifurcating parameter is the current provided by a DC current source, which will drive the equilibrium through and beyond the critical operating value $\left(v_{n}^{*}, \zeta\left(v_{n}^{*}\right)\right)$. In order to allow for a relatively simple characterization of the bifurcation phenomenon, this current source is assumed to be unique. The assumption that it defines a cutset together with the non-passive resistor and 
at least one capacitor will provide a minimal extension of the IC-cutset configuration which, as indicated above, is responsible for a null eigenvalue; this is the key assumption in Theorem 2 . The other topological conditions are meant to guarantee that the remaining conditions in Theorem 1 do hold, but they are by no means unduly restrictive. In particular, to keep the discussion in an index-one context we need a minimal extension of the topological index properties stated above; namely, implicit in our assumptions we will have the requirement that the circuit displays neither ILN-cutsets (cutsets defined by current sources, inductors and/or the non-passive resistor) nor VC-loops; note that VC-loops are a particular case of VCL-loops.

The circuit equations will be written in a semiexplicit form, specifically,

$$
\begin{aligned}
C\left(v_{c}\right) v_{c}^{\prime} & =i_{c} \\
L\left(i_{l}\right) i_{l}^{\prime} & =v_{l} \\
0 & =B_{c} v_{c}+B_{l} v_{l}+B_{g} v_{g}+B_{n} v_{n}+B_{j} v_{j}+B_{v} V \\
0 & =Q_{c} i_{c}+Q_{l} i_{l}+Q_{g} \gamma\left(v_{g}\right)+Q_{n} \zeta\left(v_{n}\right)+Q_{j} \mu+Q_{v} i_{v} .
\end{aligned}
$$

Besides the non-passive resistor and the sources, the circuit is assumed to be composed of (possibly) nonlinear capacitors (with incremental capacitance $C\left(v_{c}\right)$ ), inductors (their incremental inductance being $\left.L\left(i_{l}\right)\right)$ and strictly locally passive resistors; the latter are assumed to be governed by a voltage-controlled characteristic $i_{g}=\gamma\left(v_{g}\right)$ and, for later use, we denote by $G\left(v_{g}\right)$ the incremental conductance matrix $\gamma^{\prime}\left(v_{g}\right)$. It is worth emphasizing that the results apply also to problems with charge-controlled capacitors and flux-controlled inductors; an example will be discussed in Subsection 5.3. The current source branch has voltage $v_{j}$ (and current $\mu$ ) and the DC voltage sources branches have currents $i_{v}$ and voltages $V$. Note that the subscripts $c, l, g, n, j$ and $v$ are used to denote capacitors, inductors, passive resistors, non-passive resistors, current sources and voltage sources, not only in the branch voltages $v$ and currents $i$ but also in the splitting by columns of the (reduced) loop and cutset matrices $B, Q$, which are used to express Kirchhoff laws in the form $B v=0$ and $Q i=0$ in $(23 \mathrm{c})$ and $(23 \mathrm{~d})$. Find additional details about these (so-called branch-oriented) models in [Riaza, 2008].

We will join together different branch variables into $x=\left(v_{c}, i_{l}\right)$ and $y=\left(i_{c}, v_{l}, v_{g}, v_{n}, v_{j}, i_{v}\right)$; additionally, Theorem 2 assumes the existence of an equilibrium point which will be denoted by $\left(x^{*}, y^{*}, \mu^{*}\right)$, with $x^{*}=\left(v_{c}^{*}, i_{l}^{*}\right)$ and $y^{*}=\left(i_{c}^{*}, v_{l}^{*}, v_{g}^{*}, v_{n}^{*}, v_{j}^{*}, i_{v}^{*}\right)$; here $i_{c}^{*}=0, v_{l}^{*}=0$. We will also make use of the notation

$$
\begin{aligned}
& h(x, y, \mu)=\left(\begin{array}{c}
\left(C\left(v_{c}\right)\right)^{-1} i_{c} \\
\left(L\left(i_{l}\right)\right)^{-1} v_{l}
\end{array}\right) \\
& g(x, y, \mu)=\left(\begin{array}{c}
B_{c} v_{c}+B_{l} v_{l}+B_{g} v_{g}+B_{n} v_{n}+B_{j} v_{j}+B_{v} V \\
Q_{c} i_{c}+Q_{l} i_{l}+Q_{g} \gamma\left(v_{g}\right)+Q_{n} \zeta\left(v_{n}\right)+Q_{j} \mu+Q_{v} i_{v}
\end{array}\right),
\end{aligned}
$$

and $F=(h, g)$. Finally, $f$ will denote the map arising in (11) with $h$ and $g$ given in (24).

Theorem 2. Consider a circuit composed of the devices listed above. Fix an equilibrium point $\left(x^{*}, y^{*}, \mu^{*}\right)$ and assume that at this equilibrium the matrices $G, C$ and $L$ are positive definite, with $C$ and $L$ symmetric, and that $\zeta^{\prime}\left(v_{n}^{*}\right)=0, \zeta^{\prime \prime}\left(v_{n}^{*}\right) \neq 0$. Assume that the circuit meets the following graph-theoretic assumptions.

(1) It has a unique ICN-cutset, which includes the current source, the non-passive resistor and at least one capacitor.

(2) It exhibits neither ILN-cutsets nor VCL-loops.

Then the circuit experiences a saddle-node bifurcation as the parameter $\mu$ undergoes the critical value $\mu^{*}$.

Proof. The expressions (24) defining the maps $h$ and $g$ for the circuit model (23) yield the following form for the derivatives at equilibrium:

$$
\begin{aligned}
& h_{x}=\left(\begin{array}{ll}
0 & 0 \\
0 & 0
\end{array}\right), \quad h_{y}=\left(\begin{array}{cccccc}
C^{-1} & 0 & 0 & 0 & 0 & 0 \\
0 & L^{-1} & 0 & 0 & 0 & 0
\end{array}\right), \\
& g_{x}=\left(\begin{array}{cc}
B_{c} & 0 \\
0 & Q_{l}
\end{array}\right), g_{y}=\left(\begin{array}{cccccc}
0 & B_{l} & B_{g} & B_{n} & B_{j} & 0 \\
Q_{c} & 0 & Q_{g} G & 0 & 0 & Q_{v}
\end{array}\right),
\end{aligned}
$$


where $C$ and $L$ denote the capacitance and inductance matrices evaluated at equilibrium, and $G=\gamma^{\prime}\left(v_{g}^{*}\right)$ is the conductance matrix of passive resistors evaluated as well at the equilibrium point. In particular, the expression for $g_{y}$ shows that it is indeed a nonsingular matrix, in the light of Lemma 3 and the identities

$$
\text { ker }\left(B_{l} B_{n} B_{j}\right)=\{0\}, \operatorname{ker}\left(Q_{c} Q_{v}\right)=\{0\},
$$

which owe to the absence of ILN-cutsets and VC-loops (cf. Lemma 2); notice that VC-loops are a particular instance of VCL-loops.

The spectrum of the matrix pencil $\lambda A-B^{*}$ describes, as indicated in the proof of Theorem 1 , the eigenvalues of the matrix of partial derivatives $f_{x}$ at equilibrium. Let us first note that this matrix has a null eigenvalue with geometric multiplicity one; this is just a consequence of the fact that, according to Lemma 4 , the corank of $f_{x}$ equals that of

$$
B^{*}=\left(\begin{array}{cccccccc}
0 & 0 & C^{-1} & 0 & 0 & 0 & 0 & 0 \\
0 & 0 & 0 & L^{-1} & 0 & 0 & 0 & 0 \\
B_{c} & 0 & 0 & B_{l} & B_{g} & B_{n} & B_{j} & 0 \\
0 & Q_{l} & Q_{c} & 0 & Q_{g} G & 0 & 0 & Q_{v}
\end{array}\right) .
$$

The corank of this matrix equals in turn that of

$$
\left(\begin{array}{cccccc}
B_{c} & 0 & B_{g} & B_{n} & B_{j} & 0 \\
0 & Q_{l} & Q_{g} G & 0 & 0 & Q_{v}
\end{array}\right)
$$

again as a consequence of Lemma 4 . Because of the identities

$$
\operatorname{dim} \operatorname{ker}\left(B_{c} B_{n} B_{j}\right)=1, \operatorname{ker}\left(Q_{l} Q_{v}\right)=\{0\},
$$

the former coming from the assumed existence of a unique ICN-cutset, Lemma 3 implies that $B^{*}$ (and therefore $f_{x}$ ) has indeed corank one, so that the null eigenvalue has geometric multiplicity one, as claimed.

For the null eigenvalue to be simple, we need to prove, additionally, the condition

$$
\operatorname{ker} f_{x} \cap \operatorname{im} f_{x}=\{0\}
$$

at $\left(x^{*}, \mu^{*}\right)$ (in the sequel, all derivatives are evaluated at equilibrium without further explicit mention). To show this, we make use of the identity $h_{x}=0$ to write

$$
f_{x}=-h_{y}\left(g_{y}\right)^{-1} g_{x}
$$

Assume that $v \in \operatorname{ker} f_{x} \cap \operatorname{im} f_{x}$. Let us recast the identity $v \in \operatorname{ker} f_{x}=\operatorname{ker}\left(h_{y}\left(g_{y}\right)^{-1} g_{x}\right)$ as $w \in \operatorname{ker} h_{y}$ with $w=\left(g_{y}\right)^{-1} g_{x} v$. The expression for $h_{y}$ depicted above means that $w$ must have the form $(0, \bar{w})$, and therefore the identity $w=\left(g_{y}\right)^{-1} g_{x} v$, that is, $g_{y} w=g_{x} v$ amounts to $g_{\bar{y}} \bar{w}=g_{x} v$, where $\bar{y}$ stands for the variables $\left(v_{g}, v_{n}, v_{j}, i_{v}\right)$. In circuit-theoretic terms, this identity reads as

$$
\begin{aligned}
B_{g} w_{1}+B_{n} w_{2}+B_{j} w_{3} & =B_{c} v_{1} \\
Q_{g} G w_{1}+Q_{v} w_{4} & =Q_{l} v_{2},
\end{aligned}
$$

where $\bar{w}$ is split as $\left(w_{1}, w_{2}, w_{3}, w_{4}\right)$. Now, the orthogonality of the cut-space and the cycle-space implies that the vectors $\left(-v_{1}, 0, w_{1}, w_{2}, w_{3}, 0\right),\left(0,-v_{2}, G w_{1}, 0,0, w_{4}\right)$ must be orthogonal to each other. This yields $w_{1}^{T} G w_{1}=0$ and, because of the positive definiteness of $G$, we then have $w_{1}=0$. In particular, this simplifies (27a) to

$$
-B_{c} v_{1}+B_{n} w_{2}+B_{j} w_{3}=0
$$

and (27b) to $Q_{v} w_{4}-Q_{l} v_{2}=0$; the absence of VL-loops then yields $v_{2}=0$ (and $w_{4}=0$ ).

In turn, the assumption $v \in \operatorname{im} f_{x}$ can be rewritten, using elementary properties of Schur complements, as

$$
\left(\begin{array}{l}
v \\
0
\end{array}\right)=\left(\begin{array}{ll}
h_{x} & h_{y} \\
g_{x} & g_{y}
\end{array}\right) u
$$


for a certain vector $u$. From the expressions given in (25), this identity may be recasted as

$$
\begin{aligned}
C v_{1} & =u_{3} \\
L v_{2} & =u_{4} \\
0 & =B_{c} u_{1}+B_{l} u_{4}+B_{g} u_{5}+B_{n} u_{6}+B_{j} u_{7} \\
0 & =Q_{l} u_{2}+Q_{c} u_{3}+Q_{g} G u_{5}+Q_{v} u_{8} .
\end{aligned}
$$

Inserting (30a) into (30d) we get

$$
Q_{l} u_{2}+Q_{c} C v_{1}+Q_{g} G u_{5}+Q_{v} u_{8}=0
$$

Again, the orthogonality of the cut-space and the cycle-space implies, in light of (28) and (31), the orthogonality of the vectors $\left(-v_{1}, 0,0, w_{2}, w_{3}, 0\right)$ and $\left(C v_{1}, u_{2}, G u_{5}, 0,0, u_{8}\right)$. Therefore the identity $v_{1}^{T} C v_{1}=0$ holds and the positive definiteness of $C$ yields $v_{1}=0$. Together with the identity $v_{2}=0$ shown above we get $v=\left(v_{1}, v_{2}\right)=0$, so that the null eigenvalue of $f_{x}$ is indeed a simple one, as we aimed to show.

The absence of purely imaginary eigenvalues amounts, essentially, to a result proved in [Riaza and Tischendorf, 2007], because of the fact that the non-passive resistor may be understood to yield an opencircuit at equilibrium owing to the assumption $\zeta^{\prime}\left(v_{n}^{*}\right)=0$; note however that now there is a zero eigenvalue in the dynamics (contrary to the setting considered in [Riaza and Tischendorf, 2007]). The eigenvalueeigenvector equations for the pencil $\lambda A-B^{*}$ can be easily checked to read as

$$
\left(\begin{array}{cccccccc}
-\lambda C & 0 & I & 0 & 0 & 0 & 0 & 0 \\
0 & -\lambda L & 0 & I & 0 & 0 & 0 & 0 \\
B_{c} & 0 & 0 & B_{l} & B_{g} & B_{n} & B_{j} & 0 \\
0 & Q_{l} & Q_{c} & 0 & Q_{g} G & 0 & 0 & Q_{v}
\end{array}\right)\left(\begin{array}{c}
v_{c} \\
i_{l} \\
i_{c} \\
v_{l} \\
v_{g} \\
v_{n} \\
v_{j} \\
i_{v}
\end{array}\right)=0
$$

and amount, for non-vanishing eigenvalues, to

$$
\begin{aligned}
& 0=\lambda^{-1} B_{c} C^{-1} i_{c}+\lambda B_{l} L i_{l}+B_{g} v_{g}+B_{n} v_{n}+B_{i} v_{j} \\
& 0=Q_{c} i_{c}+Q_{l} i_{l}+Q_{g} G v_{g}+Q_{v} i_{v} .
\end{aligned}
$$

We will make use of the complex conjugate (to be denoted with a superscript ${ }^{c}$ ) of (33b), namely

$$
0=Q_{c} i_{c}^{\mathrm{c}}+Q_{l} i_{l}^{\mathrm{c}}+Q_{g} G v_{g}^{\mathrm{c}}+Q_{v} i_{v}^{\mathrm{c}} .
$$

Applying again the orthogonality of the cut and cycle spaces, in this case to the vectors arising in (33a) and (34), we get

$$
0=\lambda^{-1} i_{c}^{*} C^{-1} i_{c}+\lambda i_{l}^{*} L i_{l}+v_{g}^{*} G^{\top} v_{g}
$$

where ${ }^{*}$ stands for the conjugate transpose. The semisum of (35) and its conjugate transpose is

$$
0=\operatorname{Re}\left(\lambda^{-1}\right) i_{c}^{*} C^{-1} i_{c}+\operatorname{Re}(\lambda) i_{l}^{*} L i_{l}+v_{g}^{*} \frac{G+G^{T}}{2} v_{g} .
$$

Assume now that $\lambda$ is a non-zero, purely imaginary eigenvalue. The condition $\operatorname{Re}(\lambda)=0$ implies $\operatorname{Re}\left(\lambda^{-1}\right)=0$ and simplifies the latter equation to

$$
v_{g}^{*} \frac{G+G^{T}}{2} v_{g}=0
$$

and therefore $v_{g}=0$ since $G$ is positive definite. From (33b) and the absence of VCL-loops we then get $i_{c}=i_{l}=i_{v}=0$. In turn, this simplifies (33a) to

$$
0=B_{n} v_{n}+B_{i} v_{j}
$$

Note that the absence if ILN-cutsets precludes, in particular, IN-cutsets and therefore (38) yields $v_{n}=$ $v_{j}=0$ (cf. Lemma 2). Finally, $v_{c}=v_{l}=0$ follow from the first two eigenvalue-eigenvector equations. 
Altogether, this means that all entries in the eigenvector within (32) do vanish, which is a contradiction in terms. This way, non-vanishing purely imaginary eigenvalues are ruled out and therefore all requirements on the spectrum of $\lambda A-B^{*}$ stated in the first hypothesis of Theorem 1 are met.

We still need to prove that conditions 2 and 3 in Theorem 1 hold for the circuit model (23). The matrix displayed in (14) has in this case the form

$$
\hat{B}=\left(\begin{array}{ccccccccc}
0 & 0 & C^{-1} & 0 & 0 & 0 & 0 & 0 & 0 \\
0 & 0 & 0 & L^{-1} & 0 & 0 & 0 & 0 & 0 \\
B_{c} & 0 & 0 & B_{l} & B_{g} & B_{n} & B_{j} & 0 & 0 \\
0 & Q_{l} & Q_{c} & 0 & Q_{g} G & 0 & 0 & Q_{v} & Q_{j}
\end{array}\right)
$$

the nullity of which equals that of

$$
\left(\begin{array}{ccccccc}
B_{c} & 0 & B_{g} & B_{n} & B_{j} & 0 & 0 \\
0 & Q_{l} & Q_{g} G & 0 & 0 & Q_{v} & Q_{j}
\end{array}\right) .
$$

Following Lemma 3 , non-null entries in the kernel of this matrix come from either $\operatorname{ker}\left(B_{c} B_{n} B_{j}\right)$ or $\operatorname{ker}\left(Q_{l} Q_{v} Q_{j}\right)$. The kernel of the former is one-dimensional by hypothesis, owing to the assumed existence of a unique ICN-cutset. Regarding the second matrix, we make use of the colored branch theorem (cf. Corollary 2), according to which the existence of an ICN-cutset including (at least) the current source rules out loops defined by the current source and the remaining circuit devices; in other words, the circuit cannot exhibit IVLG-loops (in particular IVL-loops) including the current source. On the other hand, IVL-loops without the current source are VL-loops, the existence of which is precluded by the exclusion of VCL-loops. This means that $\operatorname{ker}\left(Q_{l} Q_{v} Q_{j}\right)=\{0\}$. It follows that $\operatorname{dim} \operatorname{ker} \hat{B}=1$, so that $\hat{B}$ has indeed maximal rank; this means that condition 2 of Theorem 1 holds.

In order to show that condition 3 in Theorem 1 is also met, we make use of the fact that the maximal rank condition on the matrix $\tilde{B}$ in (15) can be equivalently formulated as

$$
F^{\prime \prime}\left(x^{*}, y^{*}, \mu^{*}\right)(v, v) \notin \operatorname{im} B^{*},
$$

as indicated in Subsection 2.1. As defined above, $F$ stands for the map $(h, g)$, whereas $v$ is any nontrivial vector in the kernel of $B^{*}$. The form that $B^{*}$ takes for the circuit model (23) is depicted in (26), and proceeding as above one can check that non-vanishing vectors in the kernel meet the pattern $\left(v_{1}, 0,0,0,0, v_{6}, v_{7}, 0\right)$, with non-trivial solutions to $B_{c} v_{1}+B_{n} v_{6}+B_{j} v_{7}=0$ corresponding to the existence of an ICN-cutset; since the latter is assumed to include (in particular) the non-passive resistor, we have $v_{6} \neq 0$. Note additionally that $v_{6}$ is scalar because the non-passive resistor is unique.

Additionally, it is not difficult to see that the form for $h$ and $g$ depicted in (24) yields

$$
F^{\prime \prime}\left(x^{*}, y^{*}, \mu^{*}\right)(v, v)=\left(\begin{array}{c}
0 \\
0 \\
0 \\
Q_{n} \zeta^{\prime \prime}\left(v_{n}^{*}\right) v_{6}^{2}
\end{array}\right) .
$$

Notice that the last entry is not null, because $Q_{n}$ cannot vanish (this column matrix would only vanish if the non-passive resistor defines a self-loop, a configuration which is ruled out in circuit modelling), and both $\zeta^{\prime \prime}\left(v_{n}^{*}\right)$ and $v_{6}$ are not zero. Because of the expression shown in $(40)$, the requirement $F^{\prime \prime}\left(x^{*}, y^{*}, \mu^{*}\right)(v, v) \in$ im $B^{*}$ (cf. (39)) would amount to the existence of a solution to

$$
\left(\begin{array}{c}
0 \\
0 \\
0 \\
Q_{n} \zeta^{\prime \prime}\left(v_{n}^{*}\right) v_{6}^{2}
\end{array}\right)=\left(\begin{array}{cccccccc}
0 & 0 & C^{-1} & 0 & 0 & 0 & 0 & 0 \\
0 & 0 & 0 & L^{-1} & 0 & 0 & 0 & 0 \\
B_{c} & 0 & 0 & B_{l} & B_{g} & B_{n} & B_{j} & 0 \\
0 & Q_{l} & Q_{c} & 0 & Q_{g} G & 0 & 0 & Q_{v}
\end{array}\right)\left(\begin{array}{l}
u_{1} \\
u_{2} \\
u_{3} \\
u_{4} \\
u_{5} \\
u_{6} \\
u_{7} \\
u_{8}
\end{array}\right) .
$$


From this system one easily gets $u_{3}=0, u_{4}=0$, the remaining entries yielding a solution to

$$
\begin{aligned}
& 0=B_{c} u_{1}+B_{g} u_{5}+B_{n} u_{6}+B_{j} u_{7} \\
& 0=-Q_{n} \zeta^{\prime \prime}\left(v_{n}^{*}\right) v_{6}^{2}+Q_{l} u_{2}+Q_{g} G u_{5}+Q_{v} u_{8} .
\end{aligned}
$$

However, equation (42b) signals the existence of an NLGV-loop including the non-passive resistor; this follows from the fact that $\zeta^{\prime \prime}\left(v_{n}^{*}\right) v_{6}^{2} \neq 0$, so that $Q_{n} \in \operatorname{im}\left(Q_{l} Q_{g} Q_{v}\right)$, and Corollary 1. This is in contradiction with the assumed existence of an ICN-cutset including the non-passive resistor which, in light of the colored branch theorem (Corollary 2) rules out such a loop. Therefore, (39) also holds and this completes the proof.

\section{A bifurcation of lines of equilibria in memristive circuits}

The memory-resistor or memristor is an electronic device defined by a nonlinear charge-flux characteristic. It has been the object of much recent attention in the literature on electrical circuits and electronics, coming from the report in 2008 of the design of a nanoscale device with a memristive characteristic [Strukov et al., 2008]. The origin of the memristor can be tracked back to the work of Leon Chua in 1971, who predicted the existence of such a device for symmetry reasons [Chua, 1971]. The memristor is meant to be the fourth basic circuit element, in addition to resistors, capacitors and inductors, whose characteristics relate voltage and current, voltage and charge, and flux and current, respectively. Some recent references on this topic are [Adamatzky and Chua, 2014; Buscarino et al., 2012; Di Ventra et al., 2009; Itoh and Chua, 2008; Corinto, 2011; García-Redondo et al., 2014; Itoh and Chua, 2011, 2014; Jansen et al., 2013; Kavehei et al., 2010; Messias et al., 2010; Muthuswamy and Chua, 2010; Pershin and Di Ventra, 2011, 2012; Vourkas et al., 2015; Tetzlaff, 2014].

The characteristic of a memristor may have either a charge-controlled or a flux-controlled form. For the sake of simplicity we will focus on the latter (although analogous results hold for charge-controlled memristors), so that the device will be assumed to be defined by a characteristic

$$
q=\phi(\varphi),
$$

for a smooth map $\phi$. By differentiating this relation one gets

$$
i=W(\varphi) v
$$

with

$$
W(\varphi)=\phi^{\prime}(\varphi)
$$

being the incremental memductance. The key idea is that (44) shows that the device behaves as a resistor in which the conductance depends on $\varphi(t)=\int_{-\infty}^{t} v(\tau) d \tau$, hence the memory-resistor name. A memristor will be said to be strictly locally passive if $W(\varphi)>0$ for all $\varphi$. This condition will be assumed throughout the analysis.

As detailed below, memristors are known to yield systematically non-isolated equilibrium points (cf. [Messias et al., 2010; Riaza, 2012]). It is therefore expected that an extension of the saddle-node bifurcation theorem in the terms presented in the previous sections should result in a bifurcation of manifolds of equilibria. This is indeed the case, as detailed in what follows.

\subsection{Memristive circuit model}

We will extend the setting considered in Section 4 by assuming that the circuit includes a single fluxcontrolled, strictly locally passive memristor. This entails the need to modify the circuit model in order to 
accommodate this device, as follows:

$$
\begin{aligned}
C\left(v_{c}\right) v_{c}^{\prime} & =i_{c} \\
L\left(i_{l}\right) i_{l}^{\prime} & =v_{l} \\
\varphi_{m}^{\prime} & =v_{m} \\
0 & =B_{c} v_{c}+B_{l} v_{l}+B_{g} v_{g}+B_{m} v_{m}+B_{n} v_{n}+B_{j} v_{j}+B_{v} V \\
0 & =Q_{c} i_{c}+Q_{l} i_{l}+Q_{g} \gamma\left(v_{g}\right)+Q_{m} W\left(\varphi_{m}\right) v_{m}+Q_{n} \zeta\left(v_{n}\right)+Q_{j} \mu+Q_{v} i_{v},
\end{aligned}
$$

where the subscript $m$ is used to denote the memristor. As above, for notational simplicity we will join together certain circuit variables into $x=\left(v_{c}, i_{l}, \varphi_{m}\right), y=\left(i_{c}, v_{l}, v_{g}, v_{m}, v_{n}, v_{j}, i_{v}\right)$; again, this model takes the semiexplicit differential-algebraic form depicted in (9).

As before, equilibrium points are defined by the vanishing of the right-hand side of (45); from (45a)(45c) one gets the conditions $i_{c}=0, v_{l}=0$ and $v_{m}=0$, and from (45d)-(45e) the remaining equilibrium equations are

$$
\begin{aligned}
& 0=B_{c} v_{c}+B_{g} v_{g}+B_{n} v_{n}+B_{j} v_{j}+B_{v} V \\
& 0=Q_{l} i_{l}+Q_{g} \gamma\left(v_{g}\right)+Q_{n} \zeta\left(v_{n}\right)+Q_{j} \mu+Q_{v} i_{v} .
\end{aligned}
$$

Note that the memristive flux $\varphi_{m}$ does not enter these equations. This means that equilibrium points of these circuits can never be isolated; indeed, given an equilibrium point, by modifying $\varphi_{m}$ one unfolds it to a line of equilibria. Noteworthy, this implies that a zero eigenvalue is always present in the linearization of the circuit model (45) at any equilibrium point. Find a more detailed analysis in this regard in [Riaza, 2012]. This means that, in this context, saddle-node bifurcations cannot be directly addressed in the terms considered in Sections 3 and 4, and that a somewhat different discussion is therefore needed.

\subsection{Saddle-node bifurcations of lines of equilibria}

It is reasonable to expect that appropriate conditions extending those presented in Subsection 2.1 might provide a saddle-node bifurcation theorem of lines of equilibria in dynamical systems which systematically exhibit non-isolated equilibrium points. Broadly speaking, this would characterize the splitting of a line of equilibria into two different ones in the presence of a second zero eigenvalue. Such a general analysis of saddle-node bifurcations of equilibrium lines is beyond the scope of the present paper. We focus on the characterization this phenomenon for memristive circuit models under a simplifying working assumption which requires an introductory digression; an example illustrating this behavior can be found in Subsection 5.3 below.

We will fix an equilibrium point, and assume in our analysis that the dynamics of (45) displays an invariant hypersurface which is transversal to the equilibrium line. This is reasonable in memristive circuit models (cf. Subsection 5.3) and means that there exists a codimension-one manifold, governed by an equation of the form

$$
\alpha\left(v_{c}, i_{l}, \varphi_{m}, i_{c}, v_{l}, v_{g}, v_{m}, v_{n}, v_{j}, i_{v}\right)=0
$$

such that any initial point for the dynamics of (45) yields a trajectory which remains on this manifold; moreover, the transversality to the equilibrium line means that (locally) the manifold accommodates a single equilibrium. Analytically, the transversality condition reads as

$$
\frac{\partial \alpha}{\partial \varphi_{m}}\left(v_{c}^{*}, i_{l}^{*}, \varphi_{m}^{*}, i_{c}^{*}, v_{l}^{*}, v_{g}^{*}, v_{m}^{*}, v_{n}^{*}, v_{j}^{*}, i_{v}^{*}\right) \neq 0,
$$

a condition which locally allows one to describe the manifold as

$$
\varphi_{m}=\beta\left(v_{c}, i_{l}, i_{c}, v_{l}, v_{g}, v_{m}, v_{n}, v_{j}, i_{v}\right) .
$$

In this context an extension of Theorem 2 reads as follows.

Theorem 3. Consider a circuit with a single flux-controlled memristor and assume that the hypotheses of Theorem 2 are met at a given equilibrium. Moreover, let the memristor be strictly locally passive (i.e. 
suppose $W\left(\varphi_{m}^{*}\right)>0$ ) and assume that there exists an invariant manifold of the form (47) including the equilibrium point and verifying the transversality assumption (48).

Then, for $\mu=\mu^{*}$ the equilibrium set locally defines a line which splits into two as $\mu$ undergoes the critical value $\mu^{*}$, that is, either for $\mu>\mu^{*}$ or, respectively, for $\mu<\mu^{*}$; in the other case $\left(\mu<\mu^{*}\right.$ or, respectively, $\mu>\mu^{*}$ ) the circuit displays no equilibria.

We only sketch the proof since it parallelizes that of Theorem 2, once the dynamics is restricted to the manifold defined by (47). Indeed, the expression given in (49) makes it possible to eliminate $\varphi_{m}$ from the model (45), and then express the local dynamics on the invariant manifold (47) as

$$
\begin{aligned}
C\left(v_{c}\right) v_{c}^{\prime} & =i_{c} \\
L\left(i_{l}\right) i_{l}^{\prime} & =v_{l} \\
0 & =B_{c} v_{c}+B_{l} v_{l}+B_{g} v_{g}+B_{m} v_{m}+B_{n} v_{n}+B_{j} v_{j}+B_{v} V \\
0 & =Q_{c} i_{c}+Q_{l} i_{l}+Q_{g} \gamma\left(v_{g}\right)+Q_{m} W(\beta(\ldots)) v_{m}+Q_{n} \zeta\left(v_{n}\right)+Q_{j} \mu+Q_{v} i_{v},
\end{aligned}
$$

where we skip the arguments of $\beta$ for the sake of notational simplicity; the variables actually involved are in general those displayed in (49).

The dynamical system (50) has an isolated equilibrium which meets the saddle-node requirements compiled in Theorem 1. Indeed, at equilibrium we have $v_{m}=0$ and therefore all first order derivatives of the term $W(\beta(\ldots)) v_{m}$ in (50d) do vanish, except the derivative w.r.t. $v_{m}$ which equals the memductance $W$. This means that the matrix $B^{*}$ takes for the model (50) the form

$$
B^{*}=\left(\begin{array}{ccccccccc}
0 & 0 & C^{-1} & 0 & 0 & 0 & 0 & 0 & 0 \\
0 & 0 & 0 & L^{-1} & 0 & 0 & 0 & 0 & 0 \\
B_{c} & 0 & 0 & B_{l} & B_{g} & B_{m} & B_{n} & B_{j} & 0 \\
0 & Q_{l} & Q_{c} & 0 & Q_{g} G & Q_{m} W & 0 & 0 & Q_{v}
\end{array}\right),
$$

which has exactly the structure depicted in (26): note that we may rewrite

$$
\left(Q_{g} G Q_{m} W\right)=\left(Q_{g} Q_{m}\right)\left(\begin{array}{cc}
G & 0 \\
0 & W
\end{array}\right)
$$

and, since $W$ is also positive by hypothesis, the second factor is positive definite. Therefore, all remarks concerning condition 1 of Theorem 1 apply exactly as in the proof of Theorem 2. The same happens with condition 2 , just noticing that

$$
\hat{B}=\left(\begin{array}{cccccccccc}
0 & 0 & C^{-1} & 0 & 0 & 0 & 0 & 0 & 0 & 0 \\
0 & 0 & 0 & L^{-1} & 0 & 0 & 0 & 0 & 0 & 0 \\
B_{c} & 0 & 0 & B_{l} & B_{g} & B_{m} & B_{n} & B_{j} & 0 & 0 \\
0 & Q_{l} & Q_{c} & 0 & Q_{g} G & Q_{m} W & 0 & 0 & Q_{v} & Q_{j}
\end{array}\right),
$$

so that the reasoning above applies again.

The second order requirement expressed in condition 3 can be addressed similarly, in the terms shown in (40) and $F$ coming now from (50). Vectors in the kernel of $B^{*}$ have the same form as in the proof of Theorem 2, that is, they meet the pattern $\left(v_{1}, 0,0,0,0,0, v_{7}, v_{8}, 0\right)$ (note that there is an additional entry due to the presence of an extra column corresponding to the memristor). It is not difficult to check that now

$$
F^{\prime \prime}\left(x^{*}, y^{*}, \mu^{*}\right)(v, v)=\left(\begin{array}{c}
0 \\
0 \\
0 \\
Q_{n} \zeta^{\prime \prime}\left(v_{n}^{*}\right) v_{7}^{2}
\end{array}\right),
$$

where the last entry does not vanish. In this case (40) would yield a nontrivial solution to

$$
0=-Q_{n} \zeta^{\prime \prime}\left(v_{n}^{*}\right) v_{7}^{2}+Q_{l} u_{2}+Q_{g} G u_{5}+Q_{m} W u_{6}+Q_{v} u_{9} .
$$


Now this would correspond to an NLGMV-loop including the non-passive resistor, but this would contradict the assumed existence of an ICN-cutset including as well the non-passive resistor. The colored branch theorem and Corollary 2 apply again.

Altogether, this means that Theorem 1 applies to (50) and therefore this system has a single equilibrium which splits into two different ones as $\mu$ undergoes $\mu^{*}$. We just need to unfold such equilibria by letting $\varphi_{m}$ vary on a neighborhood of $\varphi_{m}^{*}$ in order to get the line of equilibria which bifurcates into two different ones, as claimed.

The example discussed in Subsection 5.3 below illustrates this phenomenon.

\subsection{Example}

We discuss below an example intended to illustrate the results above, not only the bifurcation phenomenon but also the ideas used in the proof of Theorem 3. It is also aimed at illustrating that the results also hold in the presence of flux-controlled inductors (or, in the dual case, charge-controlled capacitors). To this end, consider the circuit depicted on the left of Figure 1.
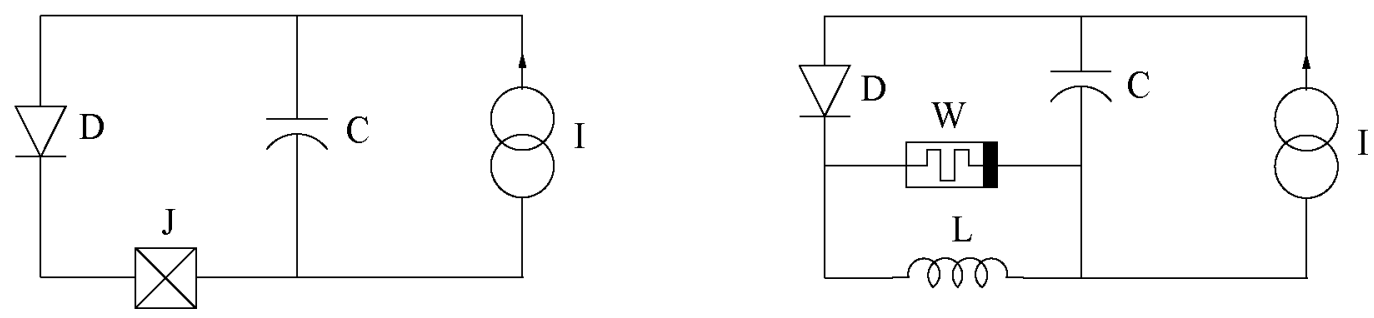

Fig. 1. (a) Example (b) Equivalent circuit.

The circuit displays a current source (labeled with an I), injecting a DC current $\mu$ : the latter will be the bifurcation parameter. Additionally, the circuit includes a linear passive capacitor (with capacitance $C$ ), a diode (labeled with a $\mathrm{D}$ ) and a Josephson junction $(\mathrm{J})$. The diode is assumed to have a smooth characteristic of the form $i_{d}=\zeta\left(v_{d}\right)$, and we will work near a local extremum located at a given $v_{d}^{*}$, describing e.g. the boundary of a tunnel effect region. To fix ideas, w.l.o.g. we will assume $v_{d}^{*}$ to be a local minimum, with $\zeta^{\prime}\left(v_{d}^{*}\right)=0, \zeta^{\prime \prime}\left(v_{d}^{*}\right)>0$.

The Josephson junction consists of two superconducting films separated by an insulating layer, yielding a nonlinear current-flux relation of the form

$$
i_{l}=I_{0} \sin \left(k_{0} \varphi_{l}\right)
$$

for certain physical constants $I_{0}, k_{0}$ : cf. [Chua et al., 1987]. For equilibria to be well-defined we need to assume that $\left|I_{0}\right|>\left|\zeta\left(v_{d}^{*}\right)\right|$. As detailed in [Chua, 2004; Jeltsema et al., 2010], realistic models of this junction should take into account not only the inductive relation (53) but also the presence of a small memristive effect (besides additional parasitic effects not relevant to our analysis). This memristive effect is well described by a flux-controlled memristor, governed by a relation of the form

$$
i_{m}=W\left(\varphi_{m}\right) v_{m}
$$

We will focus on cases in which $W$ takes on positive values. The description of the Josephson junction in terms of a parallel connection of an inductor and a memristor (governed by (53) and (54), respectively) then leads to the equivalent circuit depicted on the right of Figure 1.

The reader can easily check that the current source, the capacitor and the diode define an ICN-cutset, and that the circuit displays neither ILN-cutsets nor VCL-loops. This means that the topological conditions in Theorems 2 and 3 do hold. Additionally, we will focus on cases with positive $L, W$, so that the passivity assumptions also hold; note that $C>0$ by the aforementioned passivity assumption on the capacitor. 
Elementary circuit theory makes it possible to describe the dynamics of this circuit in terms of the DAE

$$
\begin{aligned}
C v_{c}^{\prime} & =-\zeta\left(v_{d}\right)+\mu \\
\varphi_{l}^{\prime} & =v_{c}-v_{d} \\
\varphi_{m}^{\prime}-\varphi_{l}^{\prime} & =0 \\
0 & =\zeta\left(v_{d}\right)+W\left(\varphi_{m}\right)\left(v_{d}-v_{c}\right)-I_{0} \sin \left(k_{0} \varphi_{l}\right) .
\end{aligned}
$$

Note that the flux-controlled form of the Josephson junction characteristic (53) leads naturally to a fluxoriented model.

To illustrate the bifurcation predicted by Theorem 3, we fix the parameter value $\mu^{*}=\zeta\left(v_{d}^{*}\right)$ (where, as indicated above, $v_{d}^{*}$ is assumed to be a minimum of $\zeta$ ). Equilibria of (55) for this parameter value are defined by $v_{c}=v_{d}=v_{d}^{*}$, whereas the values of $\varphi_{l}$ are defined by the identity $I_{0} \sin \left(k_{0} \varphi_{l}\right)=\zeta\left(v_{d}^{*}\right)$. Fix $\varphi_{l}^{*}$ as the (unique) solution to this equation in $\left(-\pi /\left(2 k_{0}\right), \pi /\left(2 k_{0}\right)\right)$, an interval where the incremental inductance $L$ can be easily checked to be positive. The system of equations (55) does not impose conditions on $\varphi_{m}$ for the right-hand side to vanish; this means that any vector of the form $\left(v_{c}^{*}, \varphi_{l}^{*}, \varphi_{m}, v_{d}^{*}\right)$ yields an equilibrium for this system, equilibria hence defining a line.

Since $v_{d}^{*}$ is assumed to be a local minimum of $\zeta$, (55a) already shows that there is no equilibria for $\mu<\mu^{*}$ (at least for $\mu$ close enough to $\mu^{*}$ ). By contrast, for $\mu>\mu^{*}$ (and sufficiently close to $\mu^{*}$ ) the equilibrium line indeed bifurcates into two; they are defined by the two solutions $v_{d}^{(1)}$ and $v_{d}^{(2)}$ to (55a). The values of $v_{c}$ at equilibrium are still governed by the identities $v_{c}^{(i)}=v_{d}^{(i)}(i=1,2)$ and, as before, $\varphi_{m}$ can vary freely, unfolding the two lines of equilibria. Note that $\varphi_{l}$ is uniquely defined at each equilibrium branch by the relations $I_{0} \sin \left(k_{0} \varphi_{l}^{(i)}\right)=\zeta\left(v_{d}^{(i)}\right)$ (again for $\left.i=1,2\right)$ : by continuity, these equations have a unique solution in the working interval $\left(-\pi /\left(2 k_{0}\right), \pi /\left(2 k_{0}\right)\right)$ for $\mu$ sufficiently close to $\mu^{*}$. Moreover, one can actually check that one equilibrium is stable and the other one is unstable.

To make things clearer, the reader can focus e.g. on the simple setting defined by the assumptions $\zeta\left(v_{d}\right)=v_{d}^{2}, C=I_{0}=k_{0}=1$; what follows is valid for any positive valued $W\left(\varphi_{m}\right)$ (think e.g. of $W\left(\varphi_{m}\right)=$ $\left.1+\varphi_{m}^{2}\right)$. The bifurcation occurs at $\mu^{*}=0$, with $v_{c}^{*}=v_{d}^{*}=\varphi_{l}^{*}=0$. Note that locally around these values we can explicitly eliminate $v_{d}$ from $(55 \mathrm{~d})$ as

$$
v_{d}=\frac{1}{2}\left(-W\left(\varphi_{m}\right)+\sqrt{\left(W\left(\varphi_{m}\right)\right)^{2}+4\left(W\left(\varphi_{m}\right) v_{c}+\sin \varphi_{l}\right)}\right),
$$

where we take the positive root because it defines the branch that accommodates the aforementioned equilibrium (that is, the solution $v_{d}^{*}=0$ for $v_{c}^{*}=\varphi_{l}^{*}=0$ ). Now, for $\mu<0$ the system has no equilibria, whereas for small positive $\mu$ we get $v_{d}^{(1)}=v_{c}^{(1)}=\sqrt{\mu}, v_{d}^{(2)}=v_{c}^{(2)}=-\sqrt{\mu}, \varphi_{l}^{(1)}=\arcsin (\sqrt{\mu}), \varphi_{l}^{(2)}=$ arc $\sin (-\sqrt{\mu})$; the variable $\varphi_{m}$ varies freely and parameterizes both equilibrium lines.

Our choice of the parameter values and characteristics makes it also easy to examine the stability of both equilibrium branches; skipping some simple computations, eigenvalues at both equilibria can be checked to be defined by the roots of

$$
\lambda\left(\lambda^{2}\left(2 v_{d}^{(i)}+W\right)+\lambda\left(2 v_{d}^{(i)} W+\cos \varphi_{l}^{(i)}\right)+2 v_{d}^{(i)} \cos \varphi_{l}^{(i)}\right) .
$$

As expected, there is a null eigenvalue owing to the presence of an equilibrium line; note also that $W>0$ and that $\cos \varphi_{l}^{(i)}>0$ for sufficiently small $\mu$ : this implies that the coefficients of $\lambda^{2}$ and $\lambda$ in the quadratic polynomial defining the second factor above are positive (always for small $\mu$ ), whereas the sign of the independent term equals that of $v_{d}^{(i)}$. Elementary analysis then implies that for the equilibrium branch defined by $v_{d}^{(1)}>0$ both eigenvalues are negative, whereas for $v_{d}^{(2)}<0$ there is a positive and a negative eigenvalue. This means that the first bifurcating equilibrium branch is stable, whereas the second one is unstable. This extends the well-known behavior of classical saddle-node bifurcations of equilibria in planar dynamics. 
Finally, this example makes it also possible to illustrate some of the ideas used in the proof of Theorem 3 and, specifically, the form that the invariant manifold (47) may take. Indeed, (55c) easily allows one to describe a family of invariant manifolds for the dynamics of (55), namely those described by the relations

$$
\varphi_{m}=\varphi_{l}+k
$$

for a real constant $k$. Note that the transversality condition (48) holds trivially. In each of these invariant manifolds, the reduced dynamics (which depends on $k$ ) reads as

$$
\begin{aligned}
C v_{c}^{\prime} & =-\zeta\left(v_{d}\right)+\mu \\
\varphi_{l}^{\prime} & =v_{c}-v_{d} \\
0 & =\zeta\left(v_{d}\right)+W\left(\varphi_{l}+k\right)\left(v_{d}-v_{c}\right)-I_{0} \sin \left(k_{0} \varphi_{l}\right) .
\end{aligned}
$$

In particular, fixing $\varphi_{m}^{*}$ (hence an equilibrium) and letting $k=\varphi_{m}^{*}-\varphi_{l}^{*}$ we get the invariant manifold arising in Theorem 3, (57) standing in this case for (50). Note however that is a theoretical recourse used to prove Theorem 3 in its generality, but the bifurcation of a line of equilibria in concrete cases can be described without recourse to the use of this invariant manifold, as detailed above.

\section{Concluding remarks}

This paper addresses a systematic circuit-theoretic characterization of local bifurcations in electrical and electronic circuits. The attention is focused on saddle-node bifurcations but our approach may well accommodate other phenomena such as transcritical of Hopf bifurcations. Regarding saddle-node bifurcations, we have presented an extension (of independent interest) of the theorem of Sotomayor to the context of semiexplicit index one DAEs. From the perspective of nonlinear circuit theory, bifurcations stemming from other circuit parameters, or those arising in more general settings (e.g. in distributed circuits leading to partial differential-algebraic equations (PDAEs)), may also be addressed in similar terms. A general analysis of bifurcations of manifolds of equilibria, extending the results of Section 5, is also a goal for future research.

\section{References}

A. Adamatzky and L. O. Chua (eds.), Memristor Networks, Springer, 2014.

E. L. Allgower and K. Georg, Introduction to Numerical Continuation Methods, SIAM, 2003.

B. Andrásfai, Graph Theory: Flows, Matrices, Adam Hilger, 1991.

R. E. Beardmore, The singularity-induced bifurcation and its Kronecker normal form, SIAM J. Matrix Anal. Appl. 23 (2001) 126-137.

B. Bollobás, Modern Graph Theory, Springer-Verlag, 1998.

R. K. Brayton and J. K. Moser, A theory of nonlinear networks, Quarterly of Applied Mathematics 22 (1964) 1-33, 81-104.

K. E. Brenan, S. L. Campbell and L. R. Petzold. Numerical Solution of Initial-Value Problems in Differential Algebraic Equations. SIAM, 1996.

D. P. Brown, Derivative-explicit differential equations for RLC graphs, Journal of the Franklin Institute 275 (1963) 503-514.

P. R. Bryant and J. Tow, The A-matrix of linear passive reciprocal networks, Journal of the Franklin Institute 293 (1972) 401-419.

A. Buscarino, L. Fortuna, M. Frasca and L. V. Gambuzza, A chaotic circuit based on Hewlett-Packard memristor, Chaos 22 (2012) 023136.

J. Choma Jr, Electrical Networks. Theory and Analysis, John Wiley \& Sons, New York.

L. O. Chua, Memristor - The missing circuit element, IEEE Trans. Circuit Theory 18 (1971) 507-519.

L. O. Chua, Dynamic nonlinear networks: state-of-the-art, IEEE Trans. Circ. Sys. 27 (1980) 1059-1087.

L. O. Chua, Nonlinear circuit foundations for nanodevices, Part I: The four-element torus, Proc. IEEE 91 (2004) 1830-1859.

L. O. Chua, C. A. Desoer and E. S. Kuh, Linear and Nonlinear Circuits, McGraw-Hill, 1987. 
F. Corinto, A. Ascoli and M. Gilli, Nonlinear dynamics of memristor oscillators, IEEE Trans. Circ. Sys. 58 (2011) 1323-1336.

R. Diestel, Graph Theory, Springer, 2010.

M. Di Ventra, Y. V. Pershin and L. O. Chua, Circuit elements with memory: memristors, memcapacitors and meminductors, Proc. IEEE 97 (2009) 1717-1724.

D. Estévez-Schwarz and C. Tischendorf, Structural analysis of electric circuits and consequences for MNA, Internat. J. Circuit Theory Appl. 28 (2000) 131-162.

L. Fortuna, M. Frasca and M. G. Xibilia, Chua's Circuit Implementations, World Scientific, 2009.

M. Fosséprez, Non-Linear Circuits: Qualitative Analysis of Non-Linear, Non-Reciprocal Circuits, John Wiley \& Sons, 1992.

F. R. Gantmacher, The Theory of Matrices, Chelsea, 1959.

I. García de la Vega and R. Riaza, Turning points in nonlinear circuits, Proc. Intl. Conf. on Scientific Computing in Electrical Engineering (SCEE'2014), Wuppertal, Germany, 2014 (in press).

F. García-Redondo, M. López-Vallejo and P. Ituero, Building memristor applications: From device model to circuit design, IEEE Trans. Nanotechnology 13 (2014) 1154-1162.

M. Golubitsky and V. Guillemin, Stable Mappings and Their Singularities, Springer-Verlag, 1973.

W. J. F. Govaerts, Numerical Methods for Bifurcations of Dynamical Equilibria, SIAM, 2000.

J. Guckenheimer and P. Holmes, Nonlinear Oscillations, Dynamical Systems, and Bifurcations of Vector Fields. Springer-Verlag, 1983.

M. Günther and U. Feldmann, The DAE-index in electric circuit simulation, Math. Comp. Simul. 39 (1995) $573-582$.

M. Günther and P. Rentrop, The differential-algebraic index concept in electric circuit simulation, $Z$. Angew. Math. Mech. 76 S. 1 (1996) 91-94.

B. C. Haggman and P. R. Bryant, Solutions of singular constrained differential equations: A generalization of circuits containing capacitor-only loops and inductor-only cutsets, IEEE Trans. Circ. Sys. 31 (1984) 1015-1029.

B. C. Haggman and P. R. Bryant, Geometric properties of nonlinear networks containing capacitor-only cutsets and/or inductor-only loops. Part I: Conservation laws, Cir. Sys. Signal Process. 5 (1986) 279-319.

M. Hasler and J. Neirynck, Nonlinear Circuits, Artech House, 1986.

R. A. Horn and Ch. R. Johnson, Matrix Analysis, Cambridge University Press, 1985.

M. Itoh and L. O. Chua, Memristor oscillators, Internat. J. Bifurcation and Chaos 18 (2008) 3183-3206.

M. Itoh and L. O. Chua, Memristor cellular automata and memristor discrete-time cellular neural networks, Internat. J. Bifurcation and Chaos 19 (2009) 3605-3656.

M. Itoh and L. O. Chua, Memristor Hamiltonian circuits, Internat. J. Bifurcation and Chaos 21 (2011) $2395-2425$

M. Itoh and L. O. Chua, Dynamics of memristor circuits, Internat. J. Bifurcation and Chaos 24 (2014) 1430015.

L. Jansen, M. Matthes and C. Tischendorf, Global unique solvability for memristive circuit DAEs of index 1, Int. J. Circuit Theory Appl. 43 (2015) 73-93.

D. Jeltsema and A. J. van der Schaft, Memristive port-Hamiltonian systems, Math. Comp. Model. Dyn. Sys. 16 (2010) 75-93.

O. Kavehei, A. Iqbal, Y. S. Kim, K. Eshraghian, S. F. Al-Sarawi and D. Abbott, The fourth element: characteristics, modelling and electromagnetic theory of the memristor, Proc. R. Soc. A 466 (2010) $2175-2202$.

P. Kunkel and V. Mehrmann, Differential-Algebraic Equations. Analysis and Numerical Solution, EMS, 2006.

R. Lamour, R. März and C. Tischendorf, Differential-Algebraic Equations. A Projector Based Analysis, Springer, 2013.

J. Llibre, J. Sotomayor and M. Zhitomirskii, Impasse bifurcations of constrained systems, in A. Galves et al. (eds.), Differential Equations and Dynamical Systems, Fields Inst. Commun. 31 (2002) 235-255.

R. N. Madan, Chua's Circuit: A Paradigm for Chaos, World Scientific, 1993. 
J. Martinet, Singularities of smooth functions and maps, Lecture Note Series 58, London Mathematical Society, Cambridge Univ. Press, 1982.

A. I. Mees and L. O. Chua, The Hopf bifurcation theorem and its applications to nonlinear oscillations in circuits and systems, IEEE Trans. Circ. Sys. 26 (1979) 235-254.

M. Messias, C. Nespoli and V. A. Botta, Hopf bifurcation from lines of equilibria without parameters in memristors oscillators, Internat. J. Bifurcation and Chaos 20 (2010) 437-450.

G. J. Minty, Monotone networks, Proc. Royal Soc. London A 257 (1960) 194-212.

B. Muthuswamy and L. O. Chua, Simplest chaotic circuit, Internat. J. Bifurcation and Chaos 20 (2010) $1567-1580$.

L. Perko, Differential Equations and Dynamical Systems, Springer-Verlag, 1991.

Y. V. Pershin and M. Di Ventra, Memory effects in complex materials and nanoscale systems, Advances in Physics 60 (2011) 145-227.

Y. V. Pershin and M. Di Ventra, Neuromorphic, digital and quantum computation with memory circuit elements, Proc. IEEE 100 (2012) 2071-2080.

P. J. Rabier and W. C. Rheinboldt, Theoretical and numerical analysis of differential-algebraic equations, in P. G. Ciarlet et al. (eds.), Handbook of Numerical Analysis, Vol. VIII, pp. 183-540, North Holland/Elsevier, 2002.

R. Riaza, Differential-Algebraic Systems, World Scientific, 2008.

R. Riaza, Stability loss in quasilinear DAEs by divergence of a pencil eigenvalue, SIAM J. Math. Anal. 41 (2010) 2226-2245.

R. Riaza, Manifolds of equilibria and bifurcations without parameters in memristive circuits, SIAM J. Applied Mathematics $\mathbf{7 2}$ (2012) 877-896.

R. Riaza and C. Tischendorf, Qualitative features of matrix pencils and DAEs arising in circuit dynamics, Dynamical Systems 22 (2007) 107-131.

S. Smale, On the mathematical foundations of electrical circuit theory, J. Diff. Geometry 7 (1972) 193-210.

J. Sotomayor. Structural stability and bifurcation theory, in Dynamical Systems, M. M. Peixoto (ed.). Academic Press, 549-560, 1973.

J. Sotomayor. Generic bifurcations of dynamical systems, in Dynamical Systems, M. M. Peixoto (ed.). Academic Press, 561-582, 1973.

D. B. Strukov, G. S. Snider, D. R. Stewart and R. S. Williams, The missing memristor found, Nature $\mathbf{4 5 3}$ (2008) 80-83.

R. Tetzlaff (ed.), Memristors and Memristive Systems, Springer, 2014.

C. Tischendorf, Topological index calculation of DAEs in circuit simulation, Surv. Math. Ind. 8 (1999) 187-199.

C. Tischendorf, Coupled systems of differential algebraic and partial differential equations in circuit and device simulation. Modeling and numerical analysis, Habilitationsschrift, Inst. Math., Humboldt University, Berlin, 2003.

B. Van der Pol, A theory of the amplitude of free and forced triode vibrations, Radio Rev. 1 (1920) 701-710, 754-762; Selected Scientific Papers, vol. I, North Holland, 1960, pp. 228-237, 238-246.

J. Vandewalle and L. O. Chua, The colored branch theorem and its applications in circuit theory, IEEE Trans. Circuits and Sytems 27 (1980) 816-825.

V. Venkatasubramanian, A Taxonomy of the Dynamics of Large Differential Algebraic Systems such as the Power System, PhD. Thesis, Washington University, 1992.

V. Venkatasubramanian, H. Schättler and J. Zaborszky, Local bifurcations and feasibility regions in differential-algebraic systems, IEEE Trans. Aut. Control 40 (1995) 1992-2013.

I. Vourkas, A. Batsos and C. Sirakoulis, SPICE modeling of nonlinear memristive behavior, Int. J. Circuit Theory Appl. 43 (2015) 553-565. 